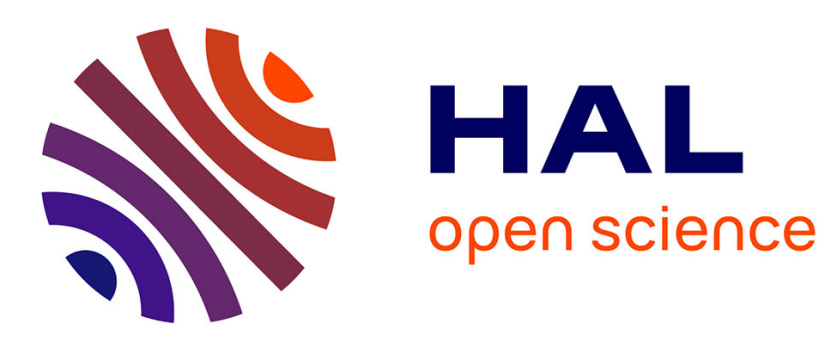

\title{
Wetting of polymers by their solvents
}

\author{
François Lequeux, Laurence Talini, Emilie Verneuil, Guillaume Delannoy, \\ Pauline Valois
}

\section{To cite this version:}

François Lequeux, Laurence Talini, Emilie Verneuil, Guillaume Delannoy, Pauline Valois. Wetting of polymers by their solvents. European Physical Journal E: Soft matter and biological physics, 2016, 39 (2), pp.12. 10.1140/epje/i2016-16012-y . hal-01281700

\section{HAL Id: hal-01281700 https://hal.sorbonne-universite.fr/hal-01281700}

Submitted on 2 Mar 2016

HAL is a multi-disciplinary open access archive for the deposit and dissemination of scientific research documents, whether they are published or not. The documents may come from teaching and research institutions in France or abroad, or from public or private research centers.
L'archive ouverte pluridisciplinaire HAL, est destinée au dépôt et à la diffusion de documents scientifiques de niveau recherche, publiés ou non, émanant des établissements d'enseignement et de recherche français ou étrangers, des laboratoires publics ou privés. 


\section{Wetting of polymers by their solvents}

François Lequeux $^{1,2}$, Laurence Talini ${ }^{1,2}$, Emilie Verneuil ${ }^{1,2}$, Guillaume Delannoy ${ }^{1,2}$ and Pauline Valois ${ }^{1,2}$.

${ }^{1}$ École Supérieure de Physique et de Chimie Industrielles de la Ville de Paris (ESPCI) ParisTech, PSL Research University, Sciences et Ingénierie de la matière Molle, CNRS UMR7615, 10, Rue Vauquelin, F-75231 Paris Cedex 05. France.

${ }^{2}$ Sorbonne-Universités, UPMC Univ. Paris 06, SIMM, 10, Rue Vauquelin, F-75231 Paris Cedex 05, France.

Abstract: We review the studies on the wetting of soluble polymeric substrates by their solvents, both in the literature and conducted in our group in the past decade. When a droplet of solvent spreads on a soluble polymer layer, its wetting angle can strongly vary with the contact line velocity even at capillary numbers smaller than unity, in contrast to non-soluble substrates. The solvent content in the polymer is a key parameter for the spreading dynamics; that content is set by the initial conditions, but also by the transfers occurring from the droplet to the polymer layer during spreading. We focus on hydrophilic amorphous polymers that are glassy at room temperature, and we discuss the consequences on wetting of the very large changes in the polymer physical properties induced by solvent sorption. We finally present new results on polymers of varying molar masses, and show how they open new perspectives for a better understanding of powder dissolution.

\section{Introduction}

Dissolution of powders is an experiment of daily life which, as everybody knows, can be difficult and result in the formation of lumps. Although frequent and of dramatic importance in several industrial processes, lump formation is a poorly understood problem. When a powder is put in contact with a liquid, capillary imbibition drives the liquid between and within grains (fig 1a). Imbibition may be heterogeneous leading to remaining dry parts in grains (fig. 1b), which results in lumps. At a smaller scale, imbibition is ruled by the advance of the contact line in a pore (fig. 1c). Understanding of how the soluble solid matter is wetted by the liquid is therefore necessary to fully prehend dissolution of powders.

In this paper we review the experimental findings on wetting of soluble substrates. We focus on the case of substrates constituted by amorphous polymers for which glass transition temperature is larger than room temperature, as for powders of amorphous polymers. When they are wetted by their solvent, rich wetting behaviours emerge, owing to the possible large variations of the polymer properties according to its solvent content. At low solvent fraction, the polymer is glassy and behaves as a rigid solid in which solvent uptake is small and solvent 
diffusion is slow, whereas at large solvent content it turns into a viscous liquid with a large solvent sorption and diffusion coefficient. Between those two situations, the polymer behaves as a soft viscoelastic medium that can easily be deformed. Such dramatic changes have important consequences on the wetting behaviour that are both described and interpreted in the following.

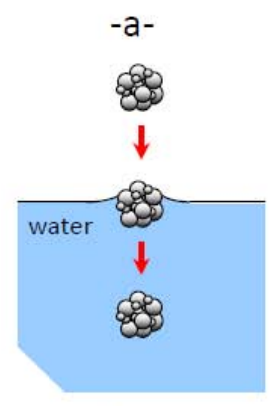

$1 \mathrm{~cm}$
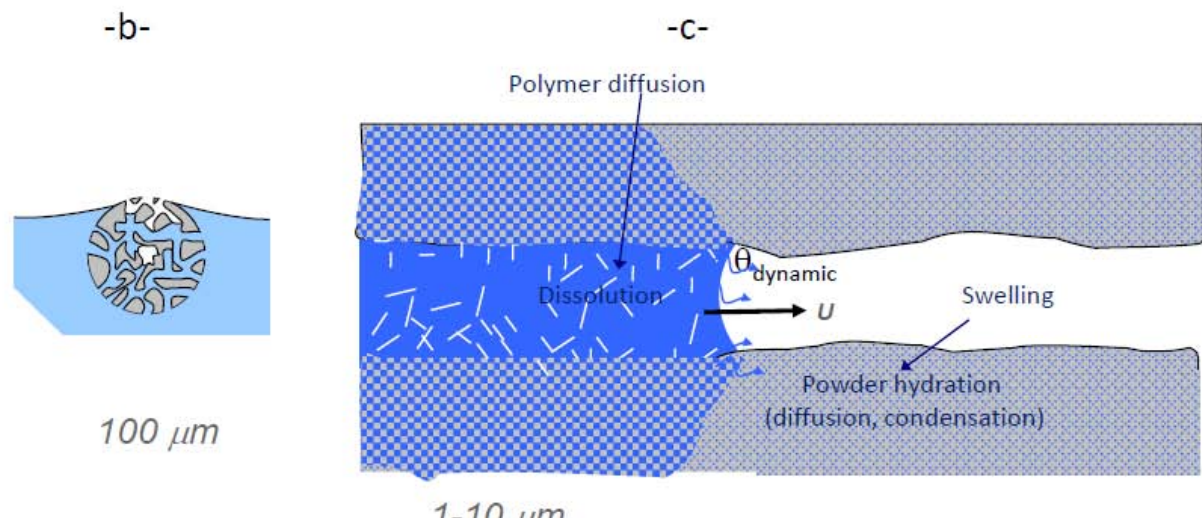

$1-10 \mu m$

Fig. 1 : Schematic representation of the dissolution process of a powder showing the grains being put in contact with the liquid (a), the incomplete imbibition of a grain by the liquid (b) and the liquid invading a pore inside a grain, with an angle $\theta$ between the contact line and the soluble solid (c). The typical lengthscales are indicated.

We will consider the simple experiment that consists in depositing a droplet of solvent on a polymer layer and in measuring both the contact angle and spreading velocity of the droplet (fig. 2). We discuss results from the literature and synthesize the results obtained in our group in the past decade, shedding new light on the ensemble of the experimental findings. We finally discuss the future works and advances needed to further understand the dissolution process of powders.

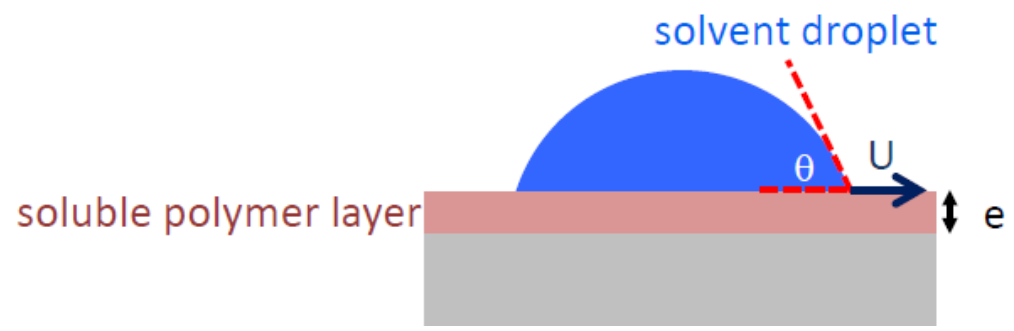

Fig 2: Schematic representation of the wetting experiment consisting in depositing a droplet of solvent on a polymer layer of thickness $e$. The contact angle $\theta$ and contact line velocity $U$ can be measured as the droplet spreads, or remains pinned on the substrate.

The paper is organised as follows: in sect. 2 we summarize the main trends of the wetting dynamics on soluble polymeric substrates and underline the solvent content in the polymer is 
a key parameter; sect. 3 is devoted to the solvent transfers occurring from the droplet to the polymer, and to their consequences on wetting dynamics. In sect. 4, we focus on glass transition effects. Finally, in sect. 5 we present new experimental data and discuss molar mass effects on the wetting dynamics. The conclusion offers perspectives, with regard to the understanding of powder dissolution.

\section{Qualitative behaviour}

The first theoretical study of the problem by Halperin and de Gennes [1] stated that "where we spread a good (non volatile) solvent on a polymer coated surface the spreading coefficient $S$ is so large that the final wetting film is usually very thin”. The statement relies on a hydration free energy becoming greater than surface energy as soon as the thickness of the layer exceeds a few monomers. In consequence, the contact angle between a polymer and its solvent is expected to be zero, which contrasts with experimental findings. Early observations actually showed that the wetting angle of a solvent on a polymer layer is finite [2]. In the case of hydrophilic polymers, that behavior was attributed to a reorientation of the polymer chains in order to expose the less hydrophilic parts at the interface with air [3]. The mechanism is driven by the minimization of the interfacial energy of the air/substrate interface. As a result, although the polymer is hydrosoluble, its surface becomes partially hydrophobic and the wetting angle can be large. The wetting of non hydrosoluble polymers has received less attention. In ref. [4], the wetting dynamics of a hydrophobic polymer (polystyrene) by a good solvent (toluene) was studied. Surprisingly, the same qualitative features as with hydrosoluble polymers and water were observed, in particular a non-zero contact angle of the solvent. The toluene content in the atmosphere was shown to influence the wetting.

The influence of the water content of the atmosphere was also studied for hydrosoluble polymers. It was reported by several authors that the angle for a hydrosoluble polymer wetted by water strongly depends on humidity $[3,5,6]$. Humidity sets the initial water content in the polymer (i.e. the content before droplet deposition), as a result the contact angle is a function of the solvent content of the polymer. That point is illustrated in fig. 3 that shows the dynamic contact angle of a water droplet spreading on a hydrosoluble polymer layer (polydimethylacrylamide), according to the initial water content of that layer. Remarkably, angles up to $90^{\circ}$ are measured, consistently with very large angle values reported in the literature [3-6]. As the water content increases, the substrate/air interfacial energy increases; consequently, the angle decreases and is expected to reach zero. It should however be noted 
that even on a gel substrate made of $80 \%$ of water or more, a water droplet can be measured to have a $50^{\circ}$ contact angle [7].

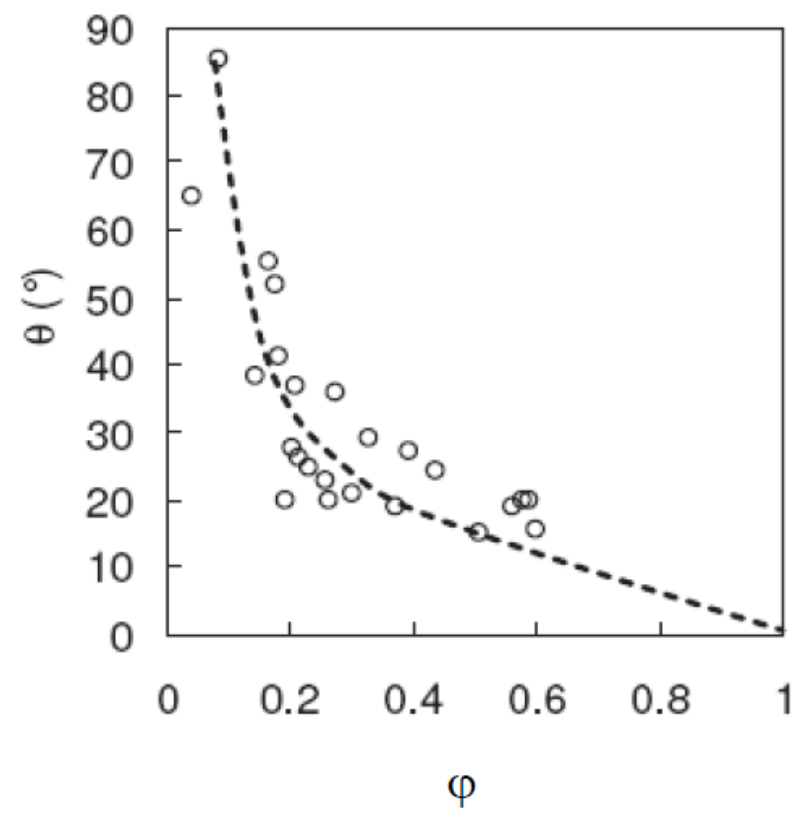

Fig. 3: Contact angle of a solvent (water) droplet as a function of the solvent volume fraction of a polymer (maltodextrine) layer, measured for a contact line velocity $U=10^{-4} \mathrm{~m} \cdot \mathrm{s}^{-1}$. The dotted line is a guide to the eye.

In addition, experiments show that, for a given humidity, the contact angle strongly varies with the contact line velocity. When a droplet spontaneously spreads on a polymer substrate, its velocity varies during the course of spreading: first, the droplet spreads fast but it further slows down. In order to investigate a wide range of velocity values, experiments in which the substrate is pulled, or the droplet is swollen can also be conducted. Velocities ranging from $10^{-6}$ to $1 \mathrm{~m} . \mathrm{s}^{-1}$ could thus be reached with water droplets on a polysaccharide (maltodextrine) substrate [8]. The variations of the contact angle measured in that range are shown in fig. 4. The angle $\theta$ is an increasing function of the contact line velocity $U$. At large velocities corresponding to early experimental times, the substrate behaves similarly as a hydrophobic one, with very large values of the wetting angle. As spreading proceeds, both the contact angle and velocity decrease with time, at a rate that decreases for vanishing values of the velocity.

The resulting $\theta$ vs. $U$ variations can be compared with the ones expected for a liquid spreading on a non soluble substrate in partial wetting conditions. In that case as well, the dynamical wetting angle depends on the contact line velocity. Considering the balance between power of the capillary driving force, $P_{f}=\gamma_{l}\left(\cos \theta_{e}-\cos \theta\right) U$, and viscous dissipation 
in the liquid droplet, $P_{d r o p}=\frac{3 \eta_{l} U^{2}}{\theta} \ln \left(\frac{L}{\kappa}\right)$ yields the Cox-Voinov law [9] for small contact angles:

$$
\left(\cos \theta_{e}-\cos \theta\right) \theta=\frac{3 \eta_{l} U}{\gamma_{l}} \ln \left(\frac{L}{\kappa}\right)
$$

Where $\theta_{e}$ is the equilibrium angle that depends on the three interfacial tensions of the problem (liquid/air, liquid/solid and solid/air), $\eta_{l}$ is the liquid viscosity and $\gamma_{l}$ the liquid/air surface tension. $L$ is the size of the liquid wedge and $\kappa$ a microscopic cut-off length. The value of $\kappa$ is widely discussed in the literature (see for instance [10]) and its order corresponds to the molecular size, leading to $\ln \left(\frac{L}{\kappa}\right) \approx 10$. However, the contact angle depends only weakly on that value: for instance $\ln \left(\frac{L}{\kappa}\right)$ varies by a factor smaller than 2 for $\frac{L}{\kappa}$ varying by a factor $10^{3}$

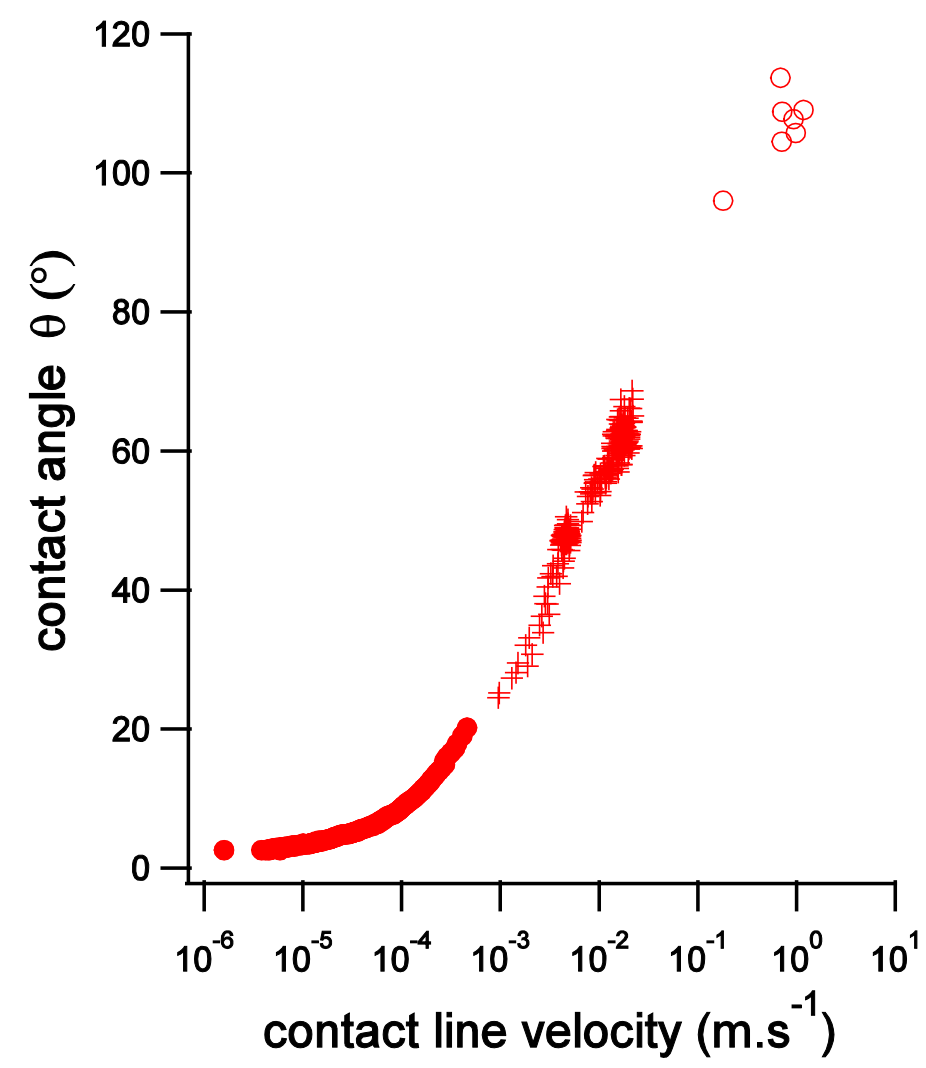

Fig. 4: Contact angle as a function of the contact line velocity for a water droplet on maltodextrine layer. To obtain the wide velocity range, experiments were conducted in three different conditions: i) spontaneous spreading (full circles), ii) droplet swollen with a pump (crosses) and iii) substrate moved at given velocity whereas the droplet remains fixed (hollow circles). 
According to eq. (1), the contact angle on a non-soluble substrate is an increasing function of the capillary number $C a=\frac{\eta_{l} U}{\gamma_{l}}$ and it is significantly larger than the equilibrium angle for $C a$ values larger than 1 . For instance, the angle increases by $5^{\circ}$ above the equilibrium angle for $C a \approx 5$. Comparatively, the range of velocities of fig. 4 corresponds to much smaller capillary numbers ranging from about $10^{-8}$ to $10^{-2}$. Therefore, the variations with velocity of the wetting angle on soluble substrates are not accounted for by the Cox-Voinov law and hence, are not due to viscous dissipation in the droplet.

As mentioned above, the dynamical wetting of soluble polymer substrates is experimentally found to be controlled by the solvent content of the polymer. In other words, $\theta=f(\varphi)$ where $\varphi$ is the solvent volume fraction at the contact line, and more precisely at a distance $\kappa$ close to the contact line. This volume fraction is determined by the initial solvent content of the polymer as described in fig. 3, but also by the solvent transfers from the droplet occurring during spreading. The transfers themselves depend on the contact line velocity. More precisely, by analogy with eq. (1), for a given liquid the wetting angle can be written as a function of the equilibrium angle, cut-off length $\kappa$ and velocity $U: \theta=f\left(\theta_{e}, \kappa, U\right)$. Note that we assume that the liquid viscosity and surface tension are not significantly modified during the wetting experiment, which is justified in the experiments we consider, that were conducted with thin layers of weakly tensioactive polymers $[8,11]$. For non soluble substrates $\theta_{e}$ and $\kappa$ have fixed values, whereas, in the case of a soluble substrate, $\theta_{e}$ and $\kappa$ become functions of the solvent content in the polymer at the contact line. Since the quantity of solvent transferred to the polymer itself depends on the velocity of the contact line, $\theta_{e}$ and $\kappa$ are implicit functions of the velocity $U$.

Solvent transfers from the droplet to the polymer layer during spreading can be evidenced with simple white light visualisations. For submicron polymer layers deposited on a reflective substrate, Newton hues can be observed, that depend on the layer thickness. As shown in fig. 5 , the hue varies in the vicinity of the contact line, evidencing the swelling of the polymer layer resulting from solvent uptake. Qualitatively, the smaller the velocity, the larger the extent of the swollen layer (see fig. $5 a$ and $5 b$ ), the quantity of solvent transferred is velocitydependent. Although the swelling can reach 50\% for nanometric films, the corresponding inclination of the substrate is very small since it takes place over very large distances (typically less than $1 \mu \mathrm{m}$ over $100 \mu \mathrm{m}$ that is less than $1 \%$ ). Note also that a vertical pulling of the substrate by the contact line would not be visible with the very thin layers that are used for 
Newton hues visualisations. In sect. 3 we discuss the transfer mechanisms at stake during spreading, and their consequences on the wetting dynamics.
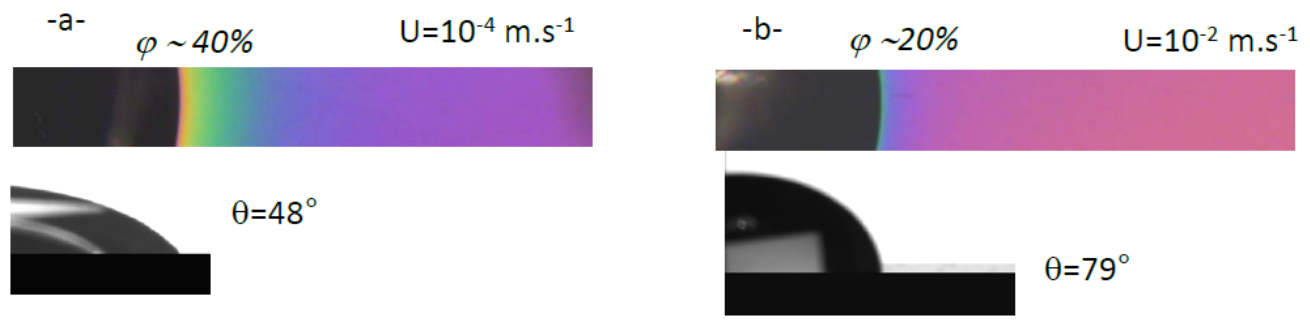

Fig. 5: Partial top and side views of a droplet spreading on a submicronic layer of polymer (polydimethylacrylamide) [3]. The variations in the Newton hues close to the contact line of the top views reflect the swelling of the polymer resulting from sorption of solvent. The photographs correspond to two different values of the contact line velocity $U=10^{-4} \mathrm{~m} \cdot \mathrm{s}^{-1}$ (a) and $U=10^{-2} \mathrm{~m} \cdot \mathrm{s}^{-1}$ (b). Smaller velocity results in a larger solvent content at the contact line, a larger extent of the solvated zone (top views) and a smaller wetting angle (side views).

\section{Solvent transfers and wetting dynamics}

As schematized in fig. 6, solvent transfers during spreading occur from i) diffusion from the droplet in the polymer layer ii) convective transportation of solvent in the polymer (the droplet moving at a velocity $U$ ) and iii) diffusion in the air of solvent evaporated from the droplet and further condensed in the polymer layer. The relative values of the fluxes associated to these three transfers depend on the distance from the contact line that is considered. For instance, at large distances from the contact line, diffusion through air is a much more efficient transfer than diffusion within the polymer. We have thus shown that the presence of the droplet modifies the water content in the atmosphere up to distances of a few hundred microns from the contact line, i.e. the macroscopic lengthscale of the problem is $L \approx 100 \mu m[12]$.

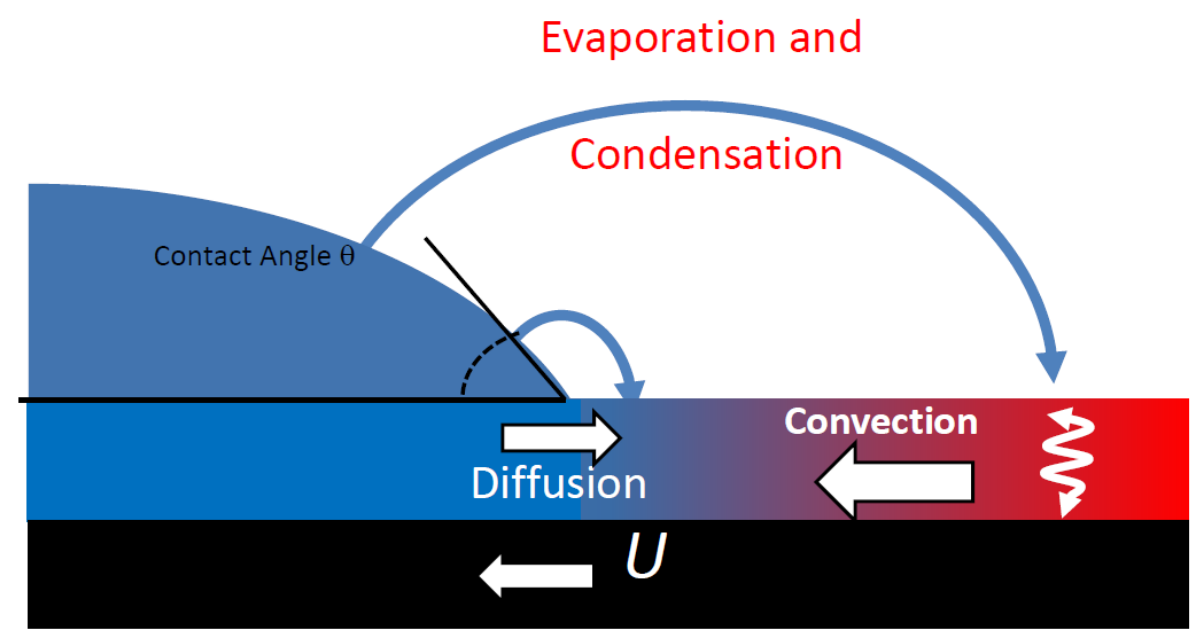


Fig. 6: Schematic representation of the transfers of solvent to the polymer layer occurring during spreading, in the reference frame of the droplet.

In the following, we denote $\varphi(r)$ the radial profile in solvent content of the polymer, where $r$ is the radial distance to the contact line. Taking all transfers into account is needed for the determination of $\varphi(r)$. However, two main difficulties arise for the solving of the equation describing the full transfer balance. The first difficulty results from the coupling of evaporation/condensation process with the other transfers. The second difficulty originates from the strongly non-linear behaviour of the polymer solvent sorption [8]: solvent uptake is small for a poorly solvated polymer, whereas it becomes large for a highly solvated polymer. As a result, the wetting dynamics exhibits a complex dependency on the initial solvent content of the polymer, but also on the thickness of the polymer layer. Figure 7a shows the angle vs. velocity of the contact line of a water droplet spreading on hydrosoluble polymer layers of different thicknesses at a given humidity. As the thickness increases, the curves are shifted towards larger wetting angles, reflecting the smaller solvent volume fraction of water transferred in the polymer for a thicker polymer layer. Two features are revealed by those curves: the first is that the wetting angle becomes independent of thickness at large layer thicknesses and velocities. The second feature is evidenced in fig. $7 \mathrm{~b}$ in which the contact angles of fig 7a are shown as a function of the quantity thickness $e$ times velocity $U$. In this representation, the data at small velocity collapse onto a master curve. Therefore, the wetting angle depends on the velocity and thickness through the product $e U$ only.
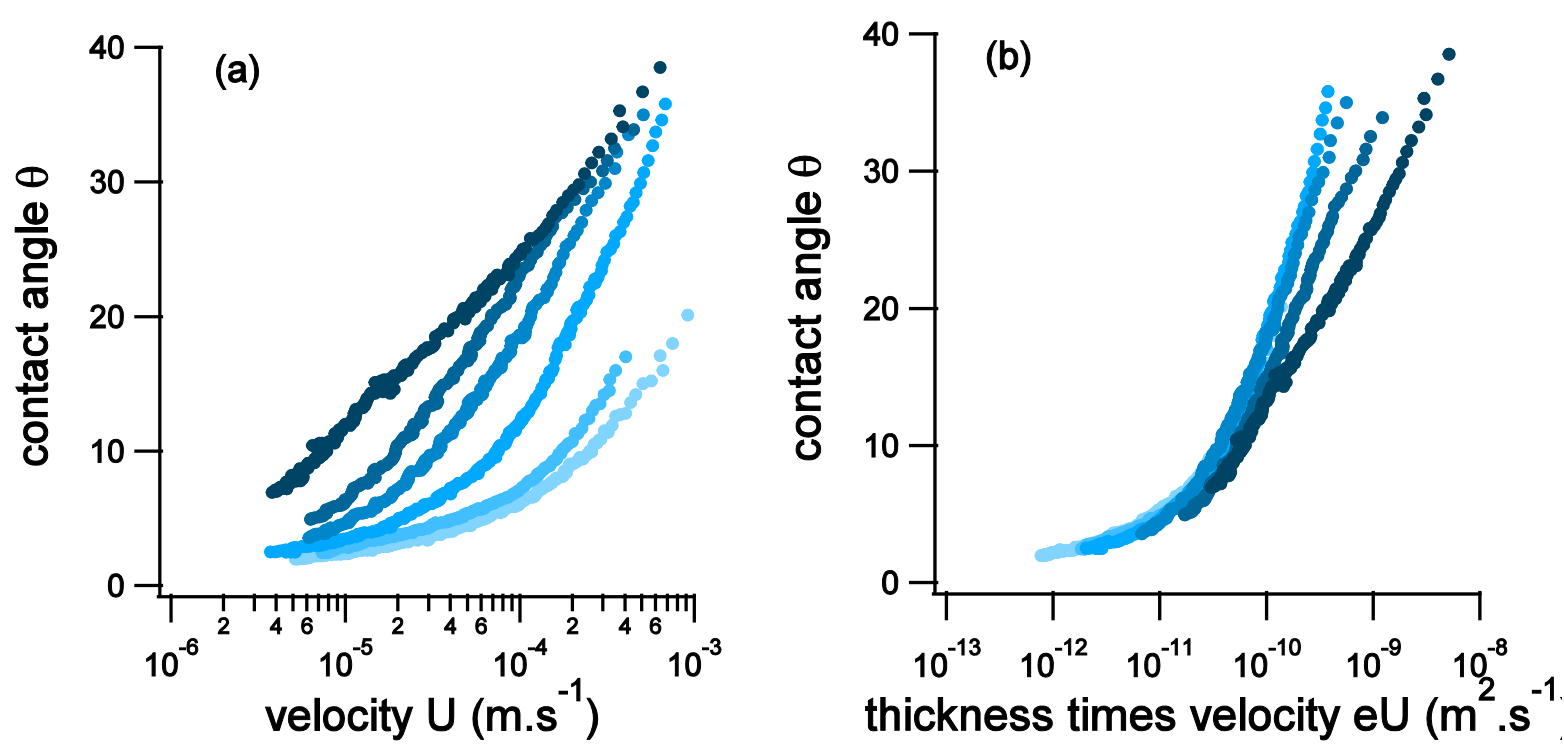
Fig. 7: Contact angle of a water droplet on a maltodextrine layer for different layer thicknesses and as a function of (a) the contact line velocity and (b) the product of layer thickness and contact line velocity. Thickness values are 100, 250, 550, 1100, 2700 and 8000 $\mathrm{nm}$ and color darkness increases with thickness.

The simple dependence in $e U$ evidenced in fig. $7 \mathrm{~b}$ at small velocities has been observed with different hydrosoluble polymers [12, 13] and is remarkably robust. It can be predicted by considering a detailed balance of the different solvent transfers during wetting [11]. Qualitative arguments can be given to understand that feature. First, the distance from the contact line relevant to the water fluxes is set by the thickness $e$ of the polymer layer. Indeed, at radial distances from the contact line smaller than $e$, the problem is a 3D problem of diffusion since convection is negligible at those scales provided the condition $e U \leq D$ is fulfilled [8], $D$ being the diffusion coefficient of water in the polymer. In particular in the latter case, the amount of water taken up by the polymer layer at distances smaller than $e$ is entirely set by the boundary condition $\varphi(r=e)$ that determines the diffusive fluxes. In particular, it does not depend on thickness $e$ and velocity $U$, or only through $\varphi(e)$. Therefore, the water volume fraction at the contact line, which determines the value of the wetting angle, is set by the value of the volume fraction at a distance $e$ from the contact line, $\varphi(e)$.

In consequence, the dependency of the wetting angle on the thickness and velocity is determined by the dependency of $\varphi(e)$ on these parameters. The way $\varphi(e)$ varies according to $e$ and $U$ results from the solvatation of the polymer layer at distances from the contact line between $e$ and the macroscopic length $L$. Within this range of distances, the most efficient solvent transfer was shown to be through the solvent evaporated in air and condensed on the layer [13]. For small velocities and/or thicknesses, the solvent transferred to the layer has time to diffuse in the vertical direction so that the solvent concentration is homogeneous along the layer thickness and over distances from the contact line ranging from $e$ to $L$. In that case, $\varphi(e)$ is given by the quantity of solvent transferred to the layer, and that quantity varies with the product $e U$ [13]. The faster the droplet, the lesser the water flux condensing on the substrate; inversely, the thicker the layer the more polymer for a given flux and the lesser the water fraction. This qualitative argument was quantitatively further developed in [13]. The condition for the layer to be homogeneous in the vertical direction is obtained by writing that the time taken by the contact line to move over a distance $e$, given by $e / U$, must be larger than the time needed for the solvent to diffuse along the layer thickness, which scales as $e^{2} / D$. In consequence, provided the condition $e U \leq D$ is satisfied, the wetting angle is a function of 
thickness and velocity through the product $e U$. In fig. $7 \mathrm{~b}$ the collapse of the $\theta$ vs. $e U$ data onto a master curve is observed for $e U \leq 10^{-10} \mathrm{~m}^{2} \cdot \mathrm{s}^{-1}$ which is in agreement with the value of the diffusion coefficient of water in the polymer.

In contrast, when the thickness is larger than $D / U$ i.e. $e U>D$, the polymer layer is not homogeneously solvated and the data does not collapse on any master curve (fig $7 \mathrm{~b}$ ). For very large thicknesses, the vertical gradient concentration within the layer extends over the region in which the water content is set by the presence of the droplet, i.e. over a distance from the contact line of the same order as the macroscopic length scale $L$ of the problem. In that case, the solvent never diffuses down to the bottom of the polymer layer, and the problem becomes thickness independent, as observed with the three thickest layers in fig. 7a.

In summary, the analysis of the different solvent transfers at stake during spreading has allowed for a description of the variations of the contact angle with the polymer thickness and contact line velocity. That result shows how the driving force for spreading is modified during the course of the wetting experiment, yielding to a velocity dependence of the wetting angle differing from the Cox-Voinov law. In the following, we show that hydration of the substrate during spreading not only modifies the substrate energy but may also give rise to a viscous dissipation in the substrate that modifies the spreading dynamics as well.

\section{Glass transition effects}

We consider amorphous polymers whose glass transition temperatures when dry are larger than room temperature. When their solvent content increases, their glass transition temperature decreases and can become smaller than room temperature, as illustrated for a hydrophilic polymer in fig. 8. The glass transition temperature decreases with increasing solvent constant, and that plasticization is well described with the empirical Fox law [14]:

$$
\frac{1}{T_{g}}=\frac{\varphi}{T_{g s}}+\frac{1-\varphi}{T_{g p}}
$$

Where $T_{g s}$ is the glass transition temperature of the solvent (water in the case of fig. 8) and $T_{g p}$ the glass transition temperature of the dry polymer. 


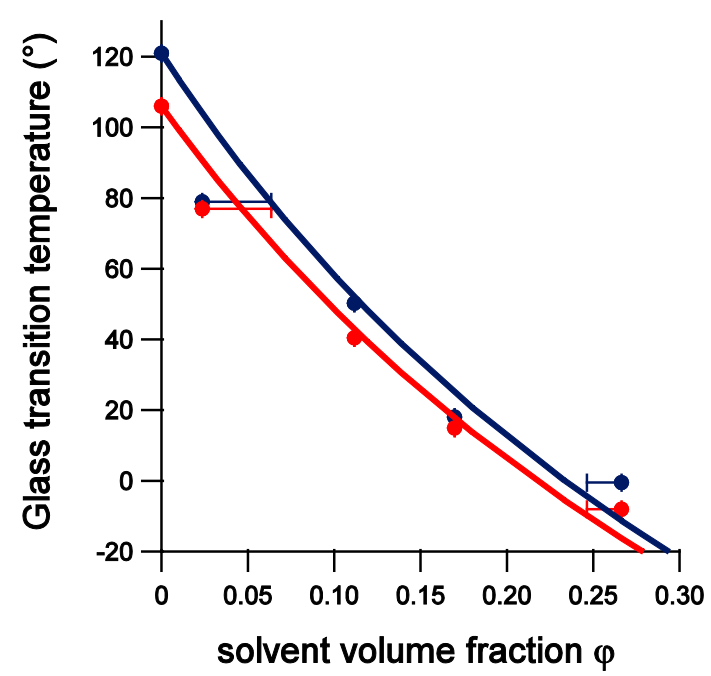

Fig. 8: Glass transition temperature as a function of water content for polydimethylacrylamide of two different molar masses (red/light gray: $54 \mathrm{~kg}_{\mathrm{mol}}{ }^{-1}$ and blue/dark gray: $1680 \mathrm{~kg} \cdot \mathrm{mol}^{-1}$ ). The experimental data (symbols) are well described with the Fox's law following equ. 2 (full lines).

Therefore, such polymers are glassy when dry, but turn in to a melt when they absorb solvent: they undergo a glass transition at constant temperature when the solvent volume fraction is increased above a value $\varphi_{g}$. In a spreading experiment in which the polymer is wetted by its solvent, an initially glassy polymer can turn into a melt during the experiment [15]. The spontaneous spreading of a droplet on such a glassy polymer layer was measured and results are reported on fig. 9a. We have found that the wetting behaviour is strongly affected with a sudden change in the angle variations with velocity that can be understood as follows: initially solvent absorption is poor and the glassy polymer has a large elastic modulus, of the order of 1GPa. In consequence, the substrate is rigid and poor hydration causes a slow decrease of both the angle and velocity as the droplet spreads. Since the capillary number is smaller than unity even at the beginning of spreading, energy is only weakly dissipated in the droplet. At this stage, the decrease in velocity and angle mainly results from solvatation of the substrate, which is poor but not zero and induces a change in the surface hydrophily. Solvent volume fraction measured from the swelling of the layer remains smaller than $\varphi_{g}$ (fig. 9b). As spreading proceeds, velocity decreases, the solvent volume fraction at the contact line increases and finally reaches $\varphi_{g}$ (fig 9c). The polymer then becomes strongly viscoelastic with an elastic modulus that decreases as the solvent content increases. As evidenced in soft materials decades ago [16], the polymer substrate deforms at the contact line under the action of the vertical component of the capillary force $\gamma_{l} \sin \theta$. Dissipation then occurs in the 
deformed zone as the contact line moves, at a much greater extent than in the liquid droplet [17]. The onset of a dissipation phenomenon leads to the change of the slope in the wetting curves. As spreading further proceeds, the substrate gets more and more hydrated, the water volume fraction is larger than $\varphi_{g}$ at macroscopic distances from the contact line. Both elastic and loss modulus of the polymer decrease for increasing hydration. The layer eventually becomes liquid like, leading to a new wetting regime. However, the transition between the two last regimes is not marked on the wetting curve and cannot be precisely identified. Glass transition induces a simultaneous change of solvent sorption, solvent coefficient diffusion and mechanical properties of the polymer, leading to steep variations of the wetting angle, whereas a smoother transition is expected as the polymer turns into a liquid.
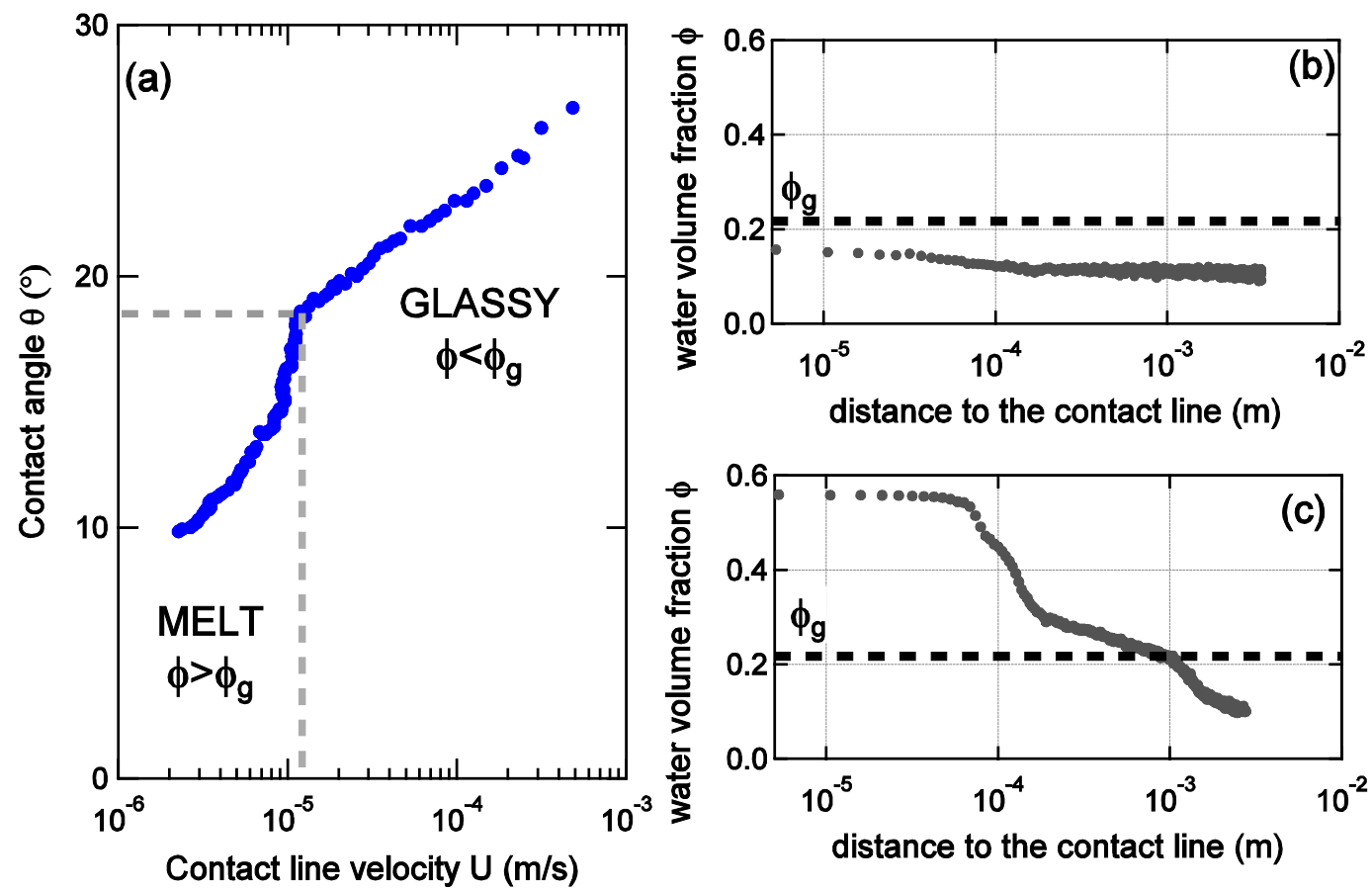

Fig. 9: Contact angle as a function of contact line velocity of a water droplet spreading on an initially glassy polymer (maltodextrine) (a). The angle variations depend on the solvent volume fraction of the polymer, which has been measured from the contact line. At high velocities the volume fraction is smaller than the critical volume fraction for glass transition $\varphi_{g}$ at all investigated distances (b) whereas at small velocities, it is larger than $\varphi_{g}$ close to the contact line and smaller than $\varphi_{g}$ farther away (c).

The universality of the observed features has been confirmed by experiments conducted with different solvents of the same polymer. The saturation concentration $C_{\text {sat }}$ in the vapour is then the parameter that most varies from one solvent to another, which changes the quantity of solvent transferred through the (most efficient) evaporation/condensation mechanism. In that 
case, the scaling in $e U$ can be more precisely expressed as a scaling in $e U\left(\varphi_{g}-\varphi_{0}\right) / D_{v} c_{\text {sat }}$, in which $D_{v}$ is the solvent diffusion coefficient in air and $\varphi_{0}$ the initial solvent volume fraction of the polymer layer. Evidence of that scaling was provided by plotting the quantity $U\left(\varphi_{g}-\varphi_{0}\right) / D_{v} c_{\text {sat }}$ measured at glass transition as a function of thickness (fig.10); in that representation, all the experimental data collapse on a master curve for different solvents of the same polymer.

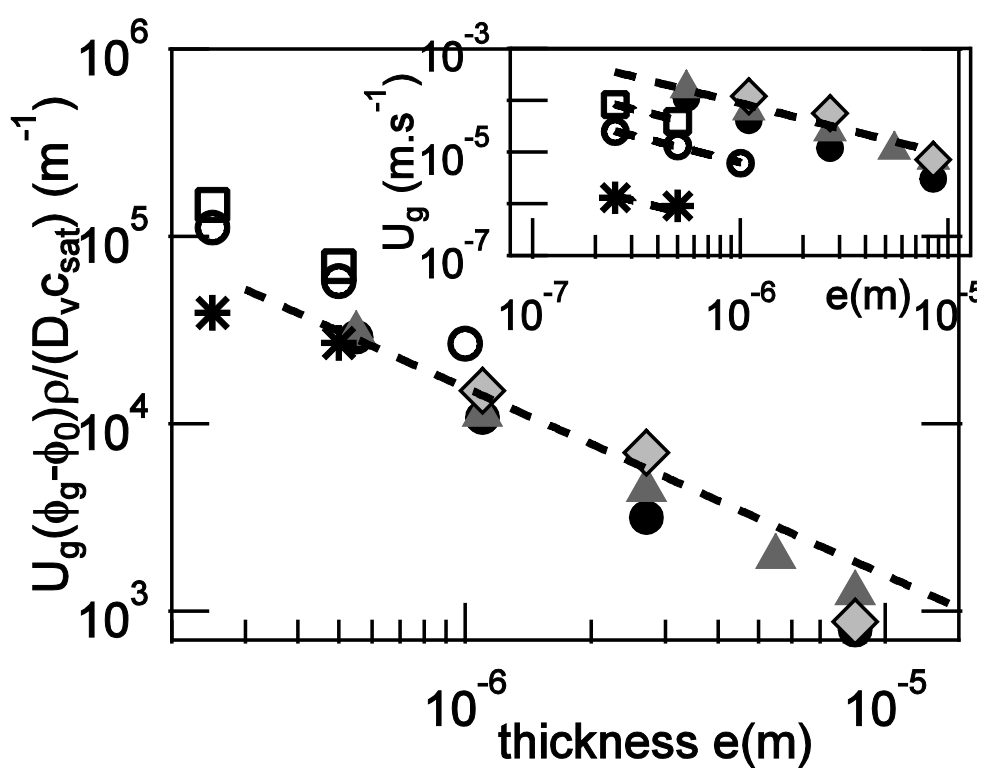

Fig. 10: Quantity $U\left(\varphi_{g}-\varphi_{0}\right) / D_{v} c_{\text {sat }}$ measured at glass transition as a function of polymer thickness for droplet of different solvents spreading on a maltodextrine layer. All data fit to $1 / e$. The figure is reproduced from ref. [15].

\section{Molar mass}

Dissolution process is known to depend on the molar mass of the polymer. However, this effect is not well understood. In the light of what precedes, molar mass is expected to affect solvent wetting dynamics since solvent sorption, but also the solvent volume fraction at which glass transition occurs, depends on molar mass. Qualitatively, $\varphi_{g}$ increases with molar mass before reaching a plateau as described by the empirical Fox law [14]. Therefore, for larger masses, glass transition is reached for larger solvent contents, as illustrated in fig. 8, in which the measured glass transition temperature of a hydrophilic polymer is reported as a function of water volume fraction for two different molar masses. Consequently, the large slope variation of the wetting curve resulting from glass transition is expected for smaller wetting angles at larger masses. Experimental observations on the wetting dynamics are in agreement with that 
behaviour: the $\theta$ vs. $U$ curves reported in fig. 11 for two different molar masses of the same polymer show that glass transition occurs at a much smaller angle for the larger mass.

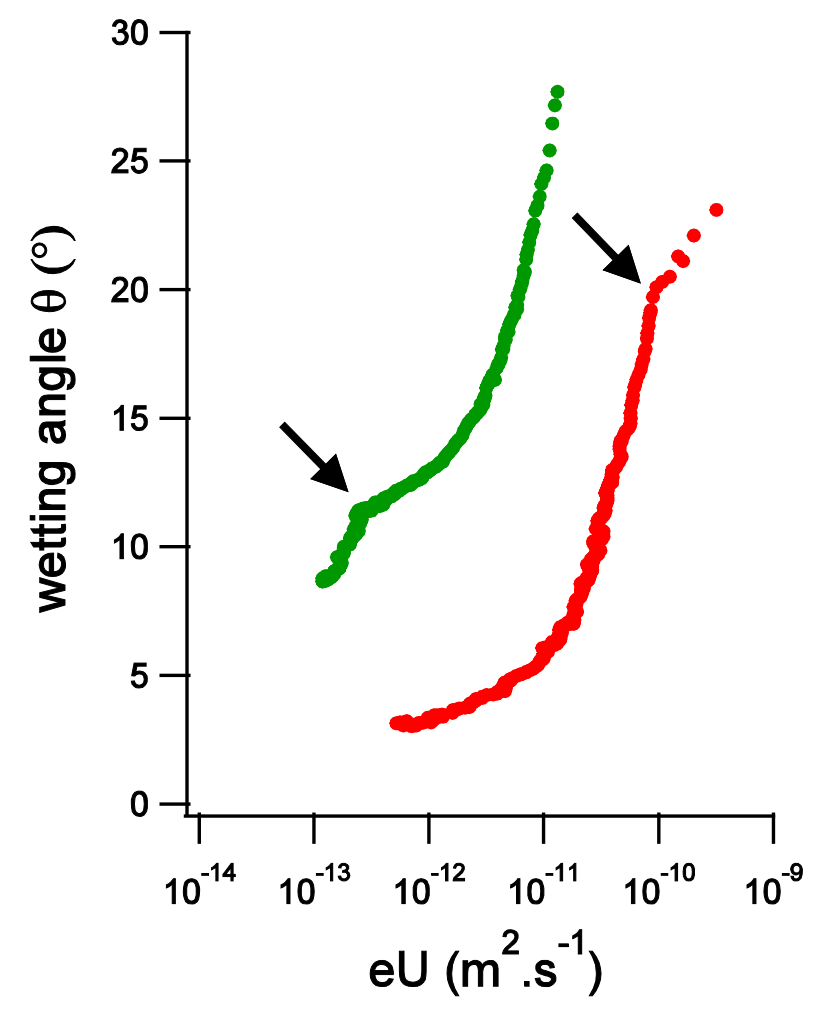

Fig. 11: Contact angle vs. velocity curves for a polymer (maltodextrine) of two different masses (red/right curve: $2.5 \mathrm{~kg} \cdot \mathrm{mol}^{-1}$, green/left curve: $80 \mathrm{~kg} \cdot \mathrm{mol}^{-1}$ ). The steep change in the slope corresponding to glass transition is shown by arrows in both cases. As expected, it occurs for smaller velocity and angle for a larger molar mass.

In addition, experiments performed in conditions for which the polymer is initially in a melt state also demonstrate a large influence of molar mass on wetting behaviour (fig. 12). Close to glass transition, the mechanical properties at a given solvent volume fraction $\varphi$ are functions of the difference $\varphi-\varphi_{g}$. A shift of the wetting curve to the smaller velocities is therefore expected when the molar mass increases, consistently with the observations.

More surprisingly, we observe that the "equilibrium" angle (i.e. the angle at vanishing velocities) also depends on the molar mass. It varies by about $10^{\circ}$ within the range of molar masses investigated in the experiments of fig. 11. At vanishing values of the velocity, the solvent content in the polymer is large and the equilibrium angle is expected to depend only on the interfacial tension with the hydrated substrate. We suggest the observed effect of molar mass on the equilibrium angle results from the influence of chain ends on the hydrophilicity of the interface with air of the polymer layer. The monomers at chain ends being more mobile than the monomers in the bulk, they are expected to reorient faster at the interface. Therefore, 
the hydrophobic parts being initially exposed at the interface with air, as the polymer is hydrated, the exposure of more hydrophilic parts is faster for small chains. Different chemical natures of the monomers of end chains (resulting from the polymerization process) may also play a part in the different values of the equilibrium angles. In the inset of fig. 11 we have reported the angles reached at $e U=10^{-14} \mathrm{~m}^{2} \cdot \mathrm{s}^{-1}$ as a function of the ratio of the volume fractions of end and bulk monomers of a polymer chain. The angle decreases before reaching a plateau as the ratio increases, in qualitative agreement with our argument. Further investigation is needed to quantitatively understand that effect.

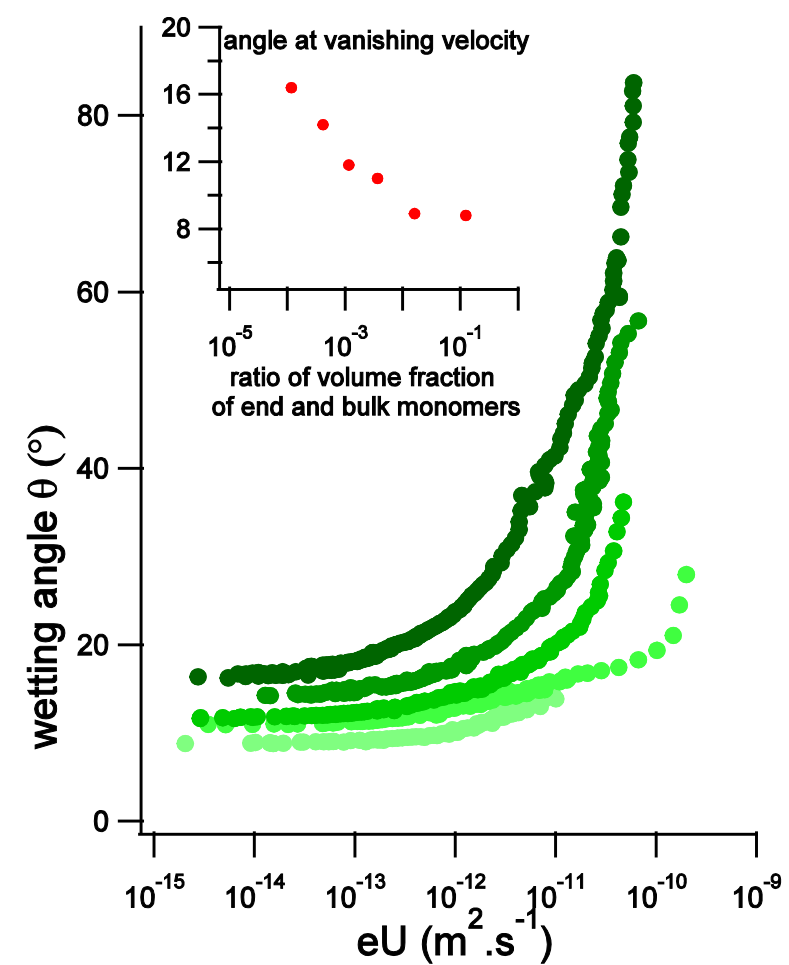

Fig. 12: Contact angle as a function of the product $e U$ for a polymer (polydimethylacrylamide) of different molar masses (from bottom to top: 12, 54, 170, 480 and $1680 \mathrm{~kg} \cdot \mathrm{mol}^{-1}$ ). The polymers are initially in a melt state. The inset shows the value of the "equilibrium" angle, i.e. the angle at vanishing velocity, as a function of the ratio of the volume fractions of end and bulk monomers.

Finally, molar mass can also influence the roughness of the surface, which is crucial in wetting experiments $[10,18]$. Polymer layers are generally formed from spin-coating or scraping of a polymer solution, and their roughness is difficult to control, in particular for low solvent content and/or polymers of large mass. Rough surfaces induce pinning of the contact line, which have been observed with polymers of different nature [4]. Interestingly, once the contact line pinned, a large swelling of the polymer layer by the contact line can be observed, as the one shown in fig. 13 for a polymer of mass larger than $10^{6}$ g.mol ${ }^{-1}$. 


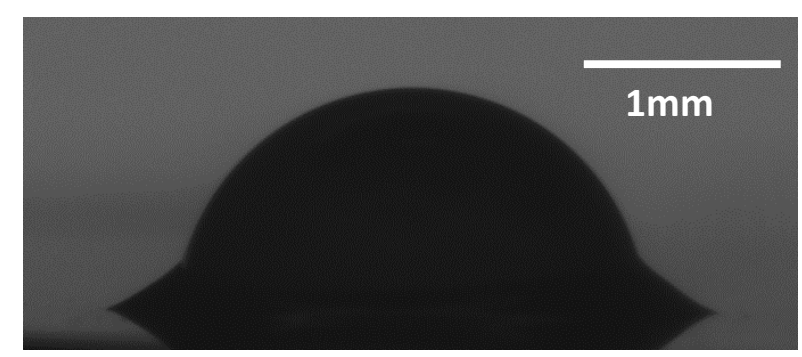

Fig. 13: Side view of a droplet pinned on a polymer layer (a high molar mass carbohydrate polymer). A polymer gel has swollen under the droplet.

Stick slip effects leading to intermittent spreading can also occur (fig. 14) with very large values of the contact angle [7]. An efficient way to smoothen the surface consists in exposing the polymer layer to an atmosphere with a large solvent content, for a time long enough for the surface to be smoothed by surface tension effects [19]. The polymer is further equilibrated in an atmosphere of lower solvent content and the wetting experiments are then reproducible, showing for instance the expected scaling with $e U$ (fig. 14).

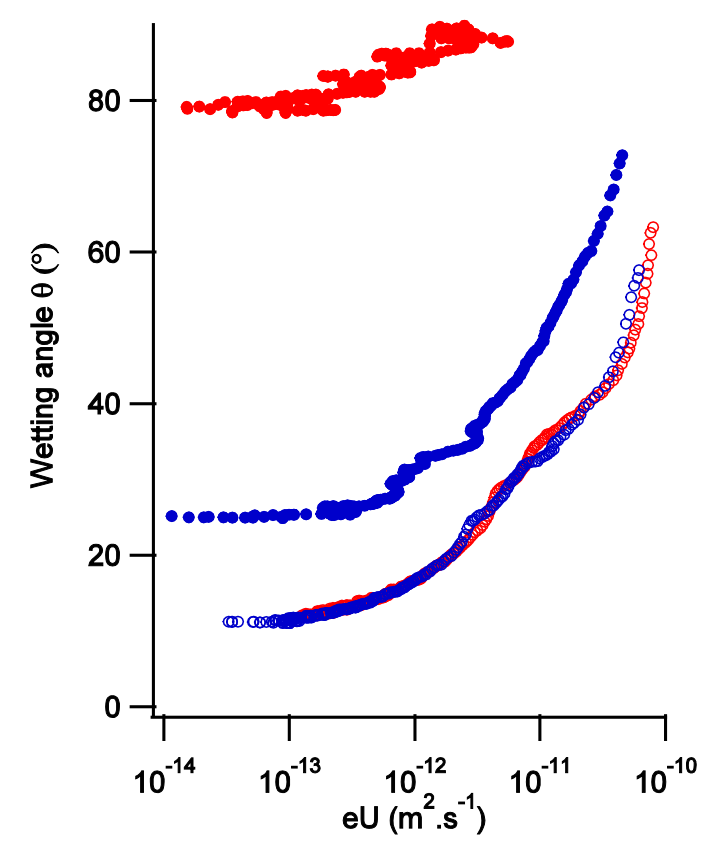

Fig. 14: Contact angle as a function of the product $e U$ for a large molar mass polymer (polydimethylacrylamide). Roughness of the layer prepared by spin coating leads to non reproducible spreading (top curves). When the layers have been smoothened, the spreading becomes reproducible and obeys the expected $e U$ scaling (two bottom curves). Blue (darker) curves correspond to a thickness of $300 \mathrm{~nm}$ and red (lighter) curves correspond to a thickness of 560nm.

For pinned drops, it is also possible to perform the wetting experiment by feeding the drops with solvents. Remarkably, the dynamics of such forced droplets exhibits the same scaling with $e U$, showing the robustness of that scaling even on rough surfaces (fig. 15). However, for 
the smaller velocities, the value of the angle towards which the curves tend at vanishing velocities is smaller than the static angle for a pinned droplet. A stick slip regime must therefore occur at vanishing values of the velocity.
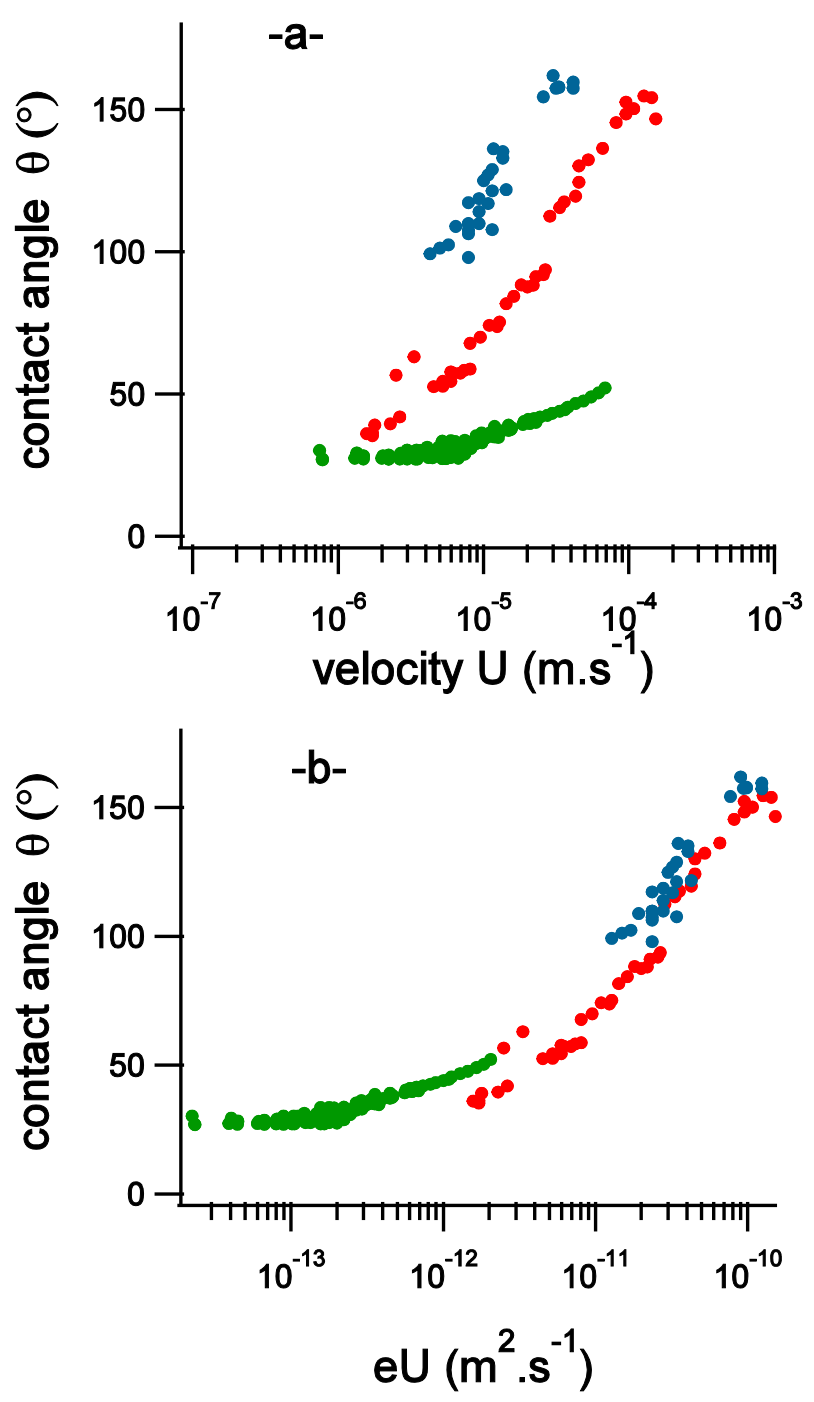

Fig. 15: Contact angle as a function of the velocity (a) and of the product $e U$ (b) for water droplets forced to spread by swelling them, and different thicknesses of the polymer (carbohydrate) layer (green: $30 \mathrm{~nm}$, red: $1 \mu \mathrm{m}$ and blue: $3 \mu \mathrm{m}$ ).

\section{Conclusion and perspectives}

In summary, the wetting dynamics of soluble substrates by volatile solvents strongly depends on the solvent content of the substrate, which is set by its initial value, but also by the transfers occurring during the course of spreading. The wetting dynamics therefore also depend on the physico-chemical properties of the polymer, such as solvent sorption, that partly determine the quantity of transferred solvent. Consequently, the contact angle exhibits a large dependence on the contact line velocity even at capillary numbers smaller than unity, i.e. for negligible viscous dissipation in the droplet. 
The velocity dependence is specifically strong in the case of polymer and solvents that are polar, since hydrophilic polymers tend to expose their less polar parts to the atmosphere. Large changes in surface energy are then observed as the polymer is hydrated. In addition, hydration induces strong modifications of the mechanical properties of the polymer with its solvent content. When the substrate is constituted by an amorphous polymer that is glassy at the temperature of the experiment, wetting dynamics is strongly affected by the mechanical behaviour or the polymer that can vary from the one of a rigid solid to the one of a viscoelastic gel and of a viscous liquid at large water content. In particular, in the range within which the polymer is viscoelastic, the wetting dynamics become strongly dissipative.

These findings are crucial for the understanding of powder dissolution. Thus, the poor dissolution of large molar mass polymers can be understood in their light. First, the larger the mass, the larger the range of solvent content for which a viscoelastic gel is formed. In addition the gel was found to be likely to pin the contact line, preventing capillary imbibitions. Second, we show that the gain in interfacial energy resulting from hydration can be smaller for large masses, owing to very long reorganisation times of the large chains. Those results therefore open the way for a better understanding of the dissolution of powders of amorphous polymers.

Author contribution statement: François Lequeux, Laurence Talini and Emilie Verneuil conceived the experiments, analyzed the data, derived the theoretical modeling and wrote the article. Guillaume Delannoy and Pauline Valois conducted experiments and analyzed data.

\section{References}

1. A. Halperin, P.G. De Gennes, J. Phys. 47, 1243 (1986).

2. F. J. Holly, Journal of Biomedical Materials Research 9, 315 (1975).

3. C. Monteux et al., Soft Matter 5, 3713 (2009).

4. $\quad$ P. Muralidhar et al., Colloid and Polymer Science 289, 1609 (2011).

5. M. J. Mackel, S. Sanchez, and J. A. Kornfield, Langmuir 23, 3 (2007).

6. K. Haraguchi, H.-J. Li, L. Song, Journal of Colloid and Interface Science 326, 41 (2008).

7. T. Kajiya et al., Soft Matter 7, 11425 (2011).

8. J. Dupas et al., Langmuir 29, 12572 (2014).

9. O.V. Voinov, Fluid Dyn. 11, 714 (1976).

10. P.G. De Gennes, Reviews of Modern Physics 57, 827 (1985). 
11. A. Tay et al., Soft Matter 7, 6953 (2011).

12. J. Dupas et al., Interfacial Phenomena and Heat Transfer 1, 231 (2013).

13. A. Tay et al., European Physical Journal E 33, 203 (2010).

14. T.G. Fox, Bull. Am. Phys. Soc. 1, 123 (1956).

15. J. Dupas et al., Physical Review Letters 112, 188302 (2014).

16. A. Carre, J. C. Gastel, M. E. R. Shanahan, Nature 379, 432 (1996).

17. D. Long, A. Ajdari, and L. Leibler, Langmuir 12, 5221 (1996).

18. S.G. Mason, Wetting, Spreading and Adhesion (Academic, New-York, 1978).

19. R. Dettre, R. Johnson in Contact Angle, Wettability and Adhesion, ed. By F.M.

Fowkes (ACS, Washington D. C., 1964). 